Int. J. Dev. Biol. 50: 143-150 (2006)

doi: $10.1387 / \mathrm{ijdb} .052042 \mathrm{bb}$

\title{
Blastula wall invagination examined on the basis of shape behavior of vesicular objects with laminar envelopes
}

\author{
BOJAN BOŽIČ 1 , JURE DERGANC ${ }^{1}$ and SAŠA SVETINA*,1,2 \\ ${ }^{1}$ Institute of Biophysics, Faculty of Medicine, University of Ljubljana, Slovenia and ${ }^{2}$ Jožef Stefan Institute, Ljubljana, Slovenia
}

\begin{abstract}
A complete description of the blastula shape transformations which occur in the process of gastrulation involves the mechanics of the blastula wall. It is inferred that the mechanical properties of this wall can, to a first approximation, be described in terms of elastic deformational modes which are equivalent to those of simple vesicular systems such as phospholipid vesicles and red blood cells. For membranes composed of an arbitrary number of aligned, laterally unconnected thin layers, these are area expansivity and local and non-local bending. Stable shapes of vesicular objects with laminar envelopes are defined as shapes which correspond to the minimum of the system elastic energy. The shape behavior of these objects is briefly reported. The criterion for the stability of the spherical shape of a vesicular object is derived. In particular we analyze the shape transition from a sphere to an invaginated gastrula. Conditions are demonstrated under which this shape transition can occur through a series of continuous shape transformations. A possible example of such a shape transformation is given. The blastula wall of the sea urchin is modeled as a closed laminar membranous system with three layers, the monocellular epithelial sheet and the extracellular apical lamina and hyaline layers. Experimentally measured elastic constants of some of these layers are shown to be in accord with the restrictions on their values imposed by the requirement for continuous shape transformations.
\end{abstract}

KEY WORDS: epithelial sheet, laminar membrane, shape transformation, gastrulation

\section{Introduction}

Shape transformations of epithelial sheets play a part in various developmental morphogenetic processes and there is thus considerable interest in elucidating the underlying mechanisms (Ettensohn, 1985; Beloussov, 1998). These mechanisms are based on the chemical and structural properties of the cells that constitute a given epithelial sheet, however, the ultimate genesis of the form of these sheets can only be understood by complementing molecular studies of the control of morphogenesis with studies of their mechanical properties (Koehl, 1990; Keller et al., 2003). Epithelial sheets often form a closed surface, such as the monocellular wall of the blastula in the development of an embryo. In the development of most embryos one of the key events is the shape transformation from the nearly spherical blastula into the invaginated gastrula. Because of its relative simplicity, the developing embryo of the sea urchin has proved to be a convenient system for studying the mechanics of such an epithelial invagination (Gustafson and Wolpert, 1967; Kominami and Takata, 2004). Several different mechanisms have been proposed to explain the shape changes in the gastrulation pro- cess (Odell et al., 1980, Ettensohn, 1985, Davidson et al., 1995, Verhas, 1997, Beloussov, 1998). Davidson et al. (1995) compared and analyzed some of them and, in the results of their more recent related measurements (Davidson et al., 1999), found support for the apical extracellular matrix of the sea urchin playing an important role, either as a mechanical structure for apicobasal contraction, a force generating structure for gel swelling, or as a substrate for cell traction.

In this work we focus on those aspects of the mechanics of the blastula wall that are related to its laminar structure. The blastula wall can be considered as a laminar structure because it involves not only a central layer constituted by the monocellular epithelial sheet but also a basolateral layer and several apical layers (Spiegel et al., 1989). Lane et al. (1993), in their gel swelling mechanism of epithelial invagination, proposed the lateral swelling of the apical lamina, caused by the cell secretion of a hygroscopic substance, to be the reason for the bending of the epithelial sheet. According to this mechanism the bending of the epithelial sheet is the consequence of the differential change of the areas of different apical layers. An analogous bilayer couple mechanism has been invoked to explain the effect of membrane

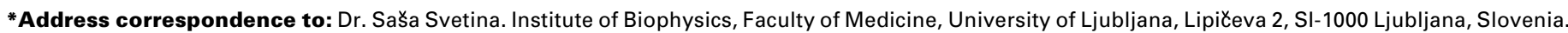
Fax: +386-1-431-5127. e-mail: sasa.svetina@biofiz.mf.uni-lj.si
} 
intercalating agents on shape transformations of red blood cells (Sheetz and Singer, 1974). A corresponding theoretical approach was developed and used to analyze the shape behavior of phospholipid vesicles and red blood cells (Svetina and Zeks, 1989, 1996). Several results of this analysis are independent of the detailed structure and composition of different laminar membranes and are therefore valid for any other vesicular object that exhibits a laminar envelope and encloses a liquid-like interior, including blastula. The notion that some intermediate shapes in the blastula - gastrula transition appear to be very similar to those observed with simple vesicular systems, such as phospholipid vesicles and red blood cells, already prompted the idea of the bilayer couple mechanism as being involved in establishing certain developmental shapes (Svetina and Zeks, 1991).

It is the purpose of this work to examine further whether some of the general conclusions obtained by analyses of the shape behavior of systems with simple laminar membranes can also contribute to understanding the shape behavior of closed epithelial sheets, in particular of the epithelial invagination in the primary step of gastrulation. We first summarize some known facts about the mechanical properties and shape behavior of simple vesicular objects that can be described in terms of the generalized bilayer couple model (Heinrich et al., 1993), frequently termed the area difference elasticity model (Miao et al., 1994). A criterion for the formation of a stable spherical shape of a vesicular object will be demonstrated. Conditions are determined under which the vesicular object can be transformed, in a continuous manner, from a sphere into an invaginated shape and a process which could give rise to such a continuous shape transformation will be presented.

We do not attempt here to give a detailed explanation of the gastrulation processes occurring in real embryos. The shape behavior of simple vesicular objects that we shall describe has to be viewed rather in the sense that it reveals the basic problems that had to be solved in the course of evolution to allow monocellular sheets to transform into invaginated shapes, which in multicellular organisms then serve in the determination of their body plan. It is plausible to assume that monocellular sheets were originally formed from identical cells and that the contemporaneous processes of epithelial invagination are upgraded versions of much simpler processes. This notion is in accord with the view (Newman and Comper, 1990) that the evolutionary origin of many morphogenetic processes may be understood in terms of the action of physical mechanisms on cells and tissues. However, in addition, the approach presented here could also contribute in revealing whether some properties of contemporary multicellular systems retain the gross features of simple vesicular systems and in distinguishing these properties from those that are the result of an evolutionary upgrading.

\section{Theoretical background}

\section{Mechanical behavior of laminar membranes}

Biological membranes, such as the red blood cell membrane, membranes of more complex eukaryotic cells and also simple phospholipid membranes, share the common feature of being composed of thin layers. In phospholipid membranes these are the two leaflets of their bilayer structure, in the red blood cell membrane the two leaflets of its bilayer part together with, in an

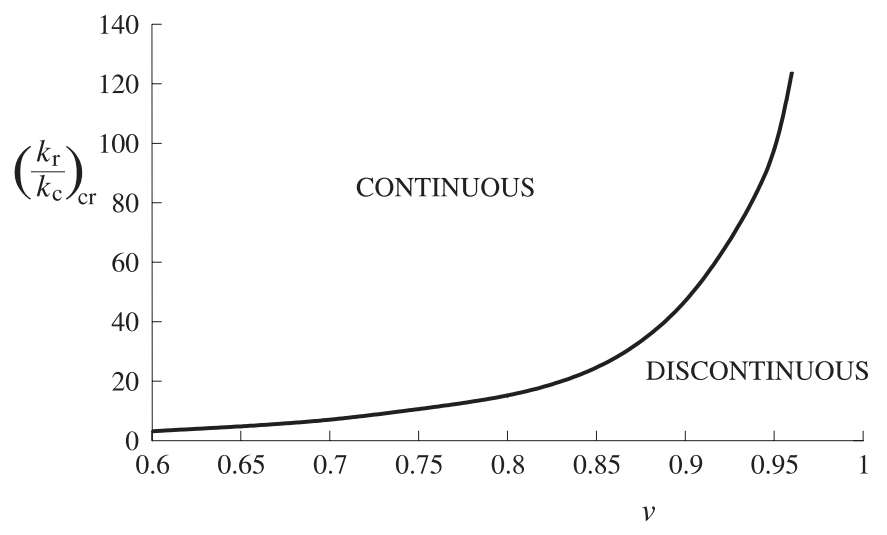

Fig. 1. The dependence of the critical value of the ratio $\left(k_{r} / k_{c}\right)_{c r}$ on the reduced volume $v$. The transition between the symmetric oblate and the cup shapes at a given $v$ is continuous only if the ratio $k_{r} / k_{c}$ is larger than the corresponding critical value. The critical ratio $\left(k_{r} / k_{c}\right)_{c r}$ is proportional to the derivative of the bending energy ( $E q .2$ by taking $C_{0}=0$ ) against the integral vesicle curvature $\bar{C}$, taken on the cup shape side of the point of the symmetry breaking transition from oblate discoid to cup shapes (see Svetina and Zeks, 2002). The bending energy of corresponding shapes was calculated numerically by the methods described in Svetina and Zeks (1989) and Seifert et al. (1991).

effective manner, the spectrin based membrane skeleton or, in the majority of eukaryotic cells, the actin rich sub-membrane cortex. These laminar systems share the characteristic property that the constituent layers are flexible but in tight contact, rendering their neutral surfaces to be equidistant over all the membrane. Frequently, the layers are not firmly connected in a manner that would prevent their lateral sliding over one another, with the consequence that mechanical relaxations in their lateral directions occur autonomously for each layer.

Mechanically, each layer of a laminar membrane exhibits bending and in-plane elasticities. The monolayers of the phospholipid bilayer, for example, are in general in a liquid crystalline state and therefore their in-plane elasticity can be described solely in terms of area expansivity (compressibility) elastic deformational mode. However, some layers of biological membranes, such as the spectrin membrane skeleton of the red blood cell, also exhibit shear elasticity. The blastula can also be considered as a vesicular object bounded by a laminar envelope. The structure of the blastula wall indicates that, to a first approximation, the mechanical properties of its constituent layers are equivalent to those of the layers of simple membranes. The central layer of the blastula wall is a monocellular sheet composed of tightly adhering epithelial cells. Cells behave, to some extent, as liquid drops (Evans and Yeung, 1989) and are thus expected, in the case of blastula shape transformations, to adapt their forms and intercellular contacts in such a way that there is no significant development of shear stresses within the layer. Other blastula wall layers, such as apical lamina, are already by nature liquidlike.

We describe now the independent deformational modes of closed laminar membranes for the case in which all the membrane layers involved are laterally liquid-like and homogeneous. In a short summary we shall present the main results of the analyses of the dependence of the elastic properties of the 
composite membrane on those of the constituent layers (Svetina and Zeks, 1992 and references therein). The area expansivity modulus of the multilayered membrane $(K)$ is the sum of the area expansivity moduli of all layers ( $K=\sum K_{i}$, with $K_{i}$ the area expansivity modulus of the $i$-th layer). The area expansivity elastic term is

$$
W_{A}=\frac{1}{2} \frac{K}{A_{0}}\left(A-A_{0}\right)^{2},
$$

where $A$ is the area and $A_{0}$ the equilibrium area of the neutral surface of the membrane. Each layer may also independently exhibit bending elasticity characterized by the bending constant $k_{c i}$ and the spontaneous curvature $C_{0 i}$. Because at a given point on the membrane all layers have equal principal curvatures $C_{1}$ and $C_{2}$, the bending energy of the whole membrane is the integral over the membrane area

$$
W_{b}=\frac{1}{2} k_{c} \int\left(C_{1}+C_{2}-C_{0}\right)^{2} d A,
$$

where the bending constant of the membrane, $k_{c}$ is the sum of the bending constants of all layers $\left(k_{c}=\sum k_{c i}\right)$ and the membrane spontaneous curvature, $C_{0}$, is the sum of the spontaneous curvatures of all layers $\left(C_{0}=\Sigma C_{0 i}\right)$.

Laminar membranes exhibit one more independent deformational mode, termed the non-local bending. It is related to the fact that, for a given shape of the vesicular object, the integral of the sum of the two principal curvatures over the whole membrane area,

$$
\bar{C}=\int\left(C_{1}+C_{2}\right) d A
$$

is not necessarily equal to its value $\left(\bar{C}_{0}\right)$ that would correspond to the shape at which the sum of area expansivity energies of all layers is minimal. Hereafter we shall denote the variable $\bar{C}$ to be the integral vesicle curvature and the parameter $\bar{C}_{0}$ the equilibrium integral vesicle curvature. The non-local bending energy arises because, at a given shape of the closed laminar membrane, all the layers, which in general have arbitrary equilibrium areas, cannot relax laterally in an optimal manner. The non-local bending energy, $W_{r}$, is given by

$$
W_{r}=\frac{1}{2} \frac{k_{r}}{A_{0}}\left(\bar{C}-\bar{C}_{0}\right)^{2}
$$

where $k_{r}$ is the non-local bending constant. The constant $k_{r}$ depends ${ }^{r}$ on the area expansivity moduli of the constituent layers $\left(K_{i}\right)$ and the distances of their neutral surfaces from that of the membrane $\left(h_{i}\right)$,

$$
k_{r}=\sum K_{i} h_{i}^{2} \text {. }
$$

The equilibrium integral vesicle curvature $\bar{C}_{0}$ for membranes with more than two layers depends on the equilibrium areas of the different membrane layers $\left(A_{0, i}\right)$, on the distances of the neutral surfaces of the layers from the neutral surface of the membrane $\left(h_{i}\right)$ and on the area expansivity moduli of the layers $\left(K_{i}\right)$. As an example we present the expression for $\bar{C}_{0}$ of an envelope composed of three layers. This expression is obtained by considering Eq. 21 in Svetina and Zeks (1992) which, for the layers i= 1,2 and 3 is:

$$
\bar{C}_{0}=\frac{K_{1} h_{1}+K_{2} h_{2}+K_{3} h_{3}}{\frac{K_{1} h_{1}^{2}}{A_{0,1}}+\frac{K_{2} h_{2}^{2}}{A_{0,2}}+\frac{K_{3} h_{3}^{2}}{A_{0,3}}},
$$

and the constraint (Eq. 16 in Svetina and Zeks, 1992),

$$
\frac{K_{1} h_{1}}{A_{0,1}}+\frac{K_{2} h_{2}}{A_{0,2}}+\frac{K_{3} h_{3}}{A_{0,3}}=0,
$$

that defines the position of the neutral surface of the trilayer membrane in terms of the two distances between the neutral surfaces of neighboring layers.

\section{Shapes of vesicular objects}

The shape of a vesicular object can be determined as that at which the expression for the elastic energy of its envelope has its minimum (Canham, 1970, Deuling and Helfrich, 1976, Svetina and Zeks, 1989, 1996). Convenient variables for characterizing possible shapes of a vesicle with volume $V$ and membrane area $A$ were found to be the reduced volume $v\left(v=V / V_{s}\right.$ where $V_{s}$ is the volume of the sphere with area $A$ ) and the reduced integral vesicle curvature $\bar{c}\left(\bar{c}=\bar{C} / \bar{C}_{s}\right.$ where $\bar{C}_{s}=8 \pi R_{s}$ is the integral vesicle curvature of the sphere with area $A=4 \pi R_{s}^{2}$ ). It is important to note that stable vesicle shapes do not exist at all allowed pairs of values of these two variables. The material constants of the system that define which shapes are stable are the reduced spontaneous curvature $c_{0}\left(c_{0}=C_{0} R_{s}\right)$, the reduced equilibrium integral vesicle curvature $\bar{c}_{0}$ (defined as $\bar{C}_{0} / \bar{C}_{s}$ ) and the ratio between the nonlocal and local bending constants $k_{r} / k_{c}$.

Analysis of the shape behavior of simple vesicular objects, such as phospholipid vesicles, revealed that there are properties of these systems that are the sole consequence of the closeness of the laminar boundary structure and that do not depend on structural and compositional details of the composing layers. The most significant general property of vesicular objects is that their shapes belong to different classes distinguished by different shape symmetries. For instance, the stomatocyte shapes and similarly invaginated gastrula shapes, belong to the class of cup

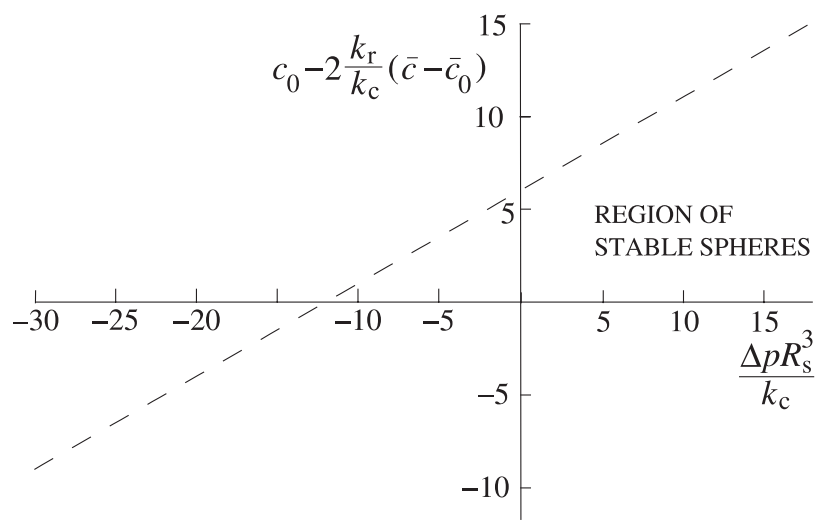

Fig. 2. The region of stable spheres in the diagram $c_{0}-2\left(k_{r} / k_{c}\right)\left(\bar{c}-\bar{c}_{0}\right)$ versus the reduced pressure difference $\Delta p R_{s}^{3} / k_{c}$. The stability condition (left side of Eq. 9 equal to zero) is presented by the dashed line. The region of stable spheres is below this line. 
shapes exhibiting a rotational symmetry (Svetina and Zeks, 1989). Another class of axisymmetric oblate shapes exhibits equatorial mirror symmetry in addition to rotational symmetry the discoid shape of the red blood cell belongs to this class. By continuously decreasing (e.g., at constant reduced volume) one or both of the parameters $c_{0}$ and $\bar{c}_{0}$, the transition from the class of symmetric oblate shapes to cup shapes can be either continuous or discontinuous, depending on the reduced volume and on the value of the ratio between the non-local and local bending constants $k_{r} / k_{c}$. For continuous shape transformations the variable $\bar{c}$ changes continuously, whereas for discontinuous transitions it exhibits a jump. Fig. 1 shows the dependence of the critical value of the ratio $\left(k_{r} / k_{c}\right)_{c r}$ on the vesicle reduced volume. The closer the vesicle volume is to that of a sphere, the larger is this ratio, attaining an infinite value at $v=1$. The occurrence of discontinuous shape transformations can be interpreted on the basis of the unique dependence of the membrane bending energy (Eq. 2) on the variables $v$ and $\bar{c}$ (Svetina and Zeks, 2002).

\section{Results}

\section{A criterion for the stability of spherical shapes}

Under certain conditions the vesicular structures defined by the energy terms Eqs. 1, 2 and 4 may attain the shape of a sphere. An equation of state (a generalized Laplace law) for this sphere was derived by considering Eqs. 1 and 2 (Ou-Yang and Helfrich, 1989). A further generalization of the equation of state, taking into account also the non-local bending energy (Eq. 4), gives

$$
\frac{2 K}{A_{0}}\left(A-A_{0}\right) R_{s}-k_{c}\left(\frac{2}{R_{s}}-C_{0}\right) C_{0} R_{s}+\frac{2 k_{r}}{A_{0}}\left(\bar{C}-\bar{C}_{0}\right)-\Delta p R_{s}^{2}=0,
$$

where $\Delta p$ is the pressure difference between the interior and exterior of the vesicular object.

Ou-Yang and Helfrich (1989) derived, by considering Eqs. 1 and 2 , the condition for the stability of the sphere. By including Eq. 4, we have generalized this condition and, in terms of the reduced variables, obtained for it the inequality

$$
c_{0}-2 \frac{k_{r}}{k_{c}}\left(1-\bar{c}_{0}\right)-\frac{\Delta p R_{s}^{3}}{2 k_{c}}-6<0 \text {. }
$$

The line that separates the region of stable spheres from the region of sphere instability in the phase diagram $c_{0}-2\left(k_{r} / k_{c}\right)\left(\bar{c}-\bar{c}_{0}\right)$ versus $\Delta p R_{s}^{3} / k_{c}$ is shown in Fig. 2. A spherical vesicular object can reach the instability line as the result of decrease of pressure difference $\Delta p$, increase of the reduced spontaneous curvature $c_{0}$, increase of the reduced equilibrium integral vesicle curvature $\bar{c}_{0}$, or of any combination of these three parameters. It is also important to note that shapes that form when the condition of sphere instability is broken are axisymmetric and exhibit equatorial mirror symmetry.

\section{A pathway for a continuous blastula-gastrula shape transfor- mation}

The shape transition of blastula into gastrula can be mimicked by a possible pathway in the $v-\bar{c}$ phase diagram through which a spherical vesicular object transforms in a continuous manner into an invaginated gastrula-like cup shape. The corresponding shape transformations are assumed to follow the shape behavior of vesicular objects with laminar envelopes that exhibit the above described mechanical properties. The two variables of the system, the reduced volume $v$ and the reduced integral vesicle curvature $\bar{c}$, have to decrease their values from the value 1 of the sphere to the values which correspond to the invaginated shape of the gastrula. The reason for choosing a continuous shape transformation process will be given in the Discussion.

The variables $v$ and $\bar{c}$ are properties of the system that, because of their different nature, have to be under the control of different cellular processes. It is plausible to expect that they change their values at different times. A possible scenario is displayed in which the chosen shape transformation pathway is presented in the $c_{0}-2\left(k_{r} / k_{c}\right)\left(\bar{c}-\bar{c}_{0}\right)$ versus $\Delta p R_{s}^{3} / k_{c}$ phase diagram (Fig. 3A) and in the $v$ versus $\bar{c}$ phase diagram (Fig. $3 \mathrm{~B})$. The first process should lead initially to instability of the spherical shape of the blastula and then to blastula flattening. We assume that initially there is a finite pressure difference between the blastula interior and exterior and that the first process causes a slow and continuous decrease of this pressure difference, while the reduced spontaneous curvature $c_{0}$ and the reduced equilibrium value of the integral vesicle curvature $\bar{c}_{0}$ remain constant. Accordingly, the process starts at point A which is within the region of stable spheres and for which the reduced integral vesicle curvature $\bar{c}=1$. In accordance with Eq. 8, the system then follows (see Fig. 3A) the line parallel to the abscissa until the instability line is reached (point B). As already stated, after crossing this line, the resulting nonspherical shapes are oblate and exhibit equatorial mirror symmetry. Making the arbitrary assumption that the pressure difference decreases continuously until it becomes zero, the reduced volume then decreases from point $B$ in Fig. $3 A$ to point C. Fig. 3B shows this part of the shape transformation pathway in the $v-\bar{c}$ phase diagram. The shape transformations are also illustrated by a sphere at the beginning (shape 1) and by two increasingly flattened shapes (shapes 2 and 3). Shape 3 is the last shape reached through the first shape sequence.

In order to achieve the final invaginated shape of the gastrula, the next process must involve a decrease of the variable $\bar{c}$. This can be accomplished by any mechanism that causes the parameter $\bar{c}_{0}$ to decrease. In Fig. 3B the consequences of this are shown for the shape transformations at constant reduced volume. At first the vesicular object still has equatorial mirror symmetry, whereas its shape is flattening. At point $D$ (see Fig. 3A and shape 4 in Fig. 3B) the equatorial mirror symmetry breaks down. At still lower values of $\bar{c}$, invagination is initiated (shape 5) which, by a further decrease of this variable, becomes deeper and deeper (shapes 6 to 8 ).

For the shape transformation at point $D$ to be continuous, the transformation pathway has to cross the line $L$ in Fig. 3B where there is a continuous, symmetry breaking transition from the oblate shapes that involve equatorial mirror symmetry into shapes that lack this symmetry. It is crucial that the ratio between the non-local and the local bending constants is larger than its critical value for the chosen reduced volume (see Fig. 1). 
A
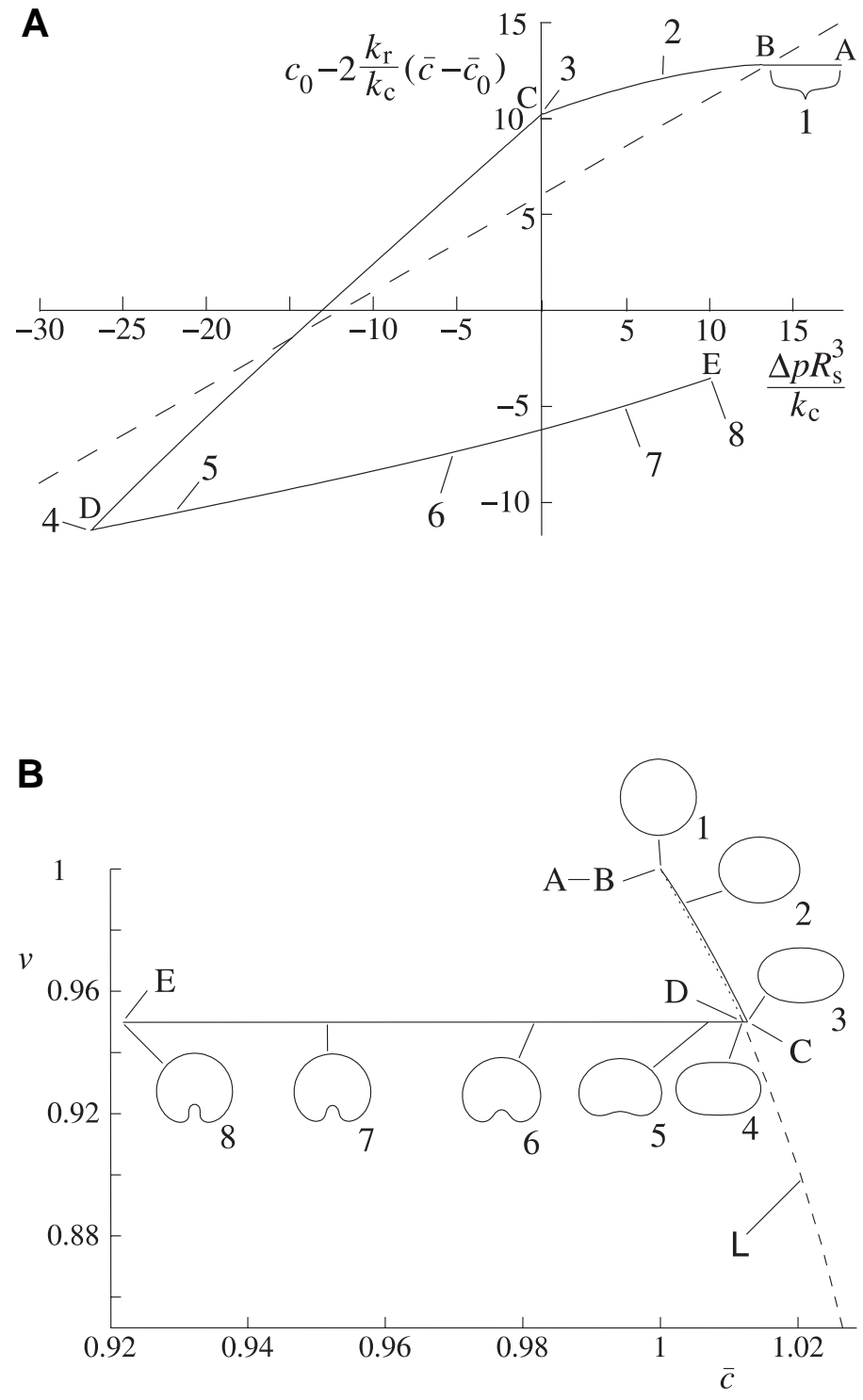

Fig. 3. A possible continuous pathway of the blastula-gastrula shape transformation. (A) The $c_{0}-2\left(k_{r} / k_{c}\right)\left(\bar{c}-\bar{c}_{0}\right)$ versus $\Delta p R_{s}^{3} / k_{c}$ phase diagram. (B) The $v$ versus $\bar{c}$ phase diagram. The pathway in (A) is denoted by thin lines. The dashed line is the instability line presented in Fig. 2. The numbers indicate the points which correspond to the shapes shown in $(B)$. In $(B)$, the line $L$ is the line of the instability of the oblate shapes exhibiting equatorial mirror symmetry. The results are presented for a value of the ratio between the non-local and local bending constants $k_{r} / k_{c}=100$ and for a reduced spontaneous curvature $c_{0}=2$. For these values of the ratio $k_{r} / k_{c}$, the shape transformation from symmetric oblate to cup shapes is continuous for the volumes for which line $L$ is plotted as a dashed line. The dotted part of line $L$ indicates that at corresponding reduced volumes, the transition from symmetric oblate to cup shapes is discontinuous. Some intermediate shapes (2-7) of the presented continuous shape transformation are also given. Shapes 1-3 are calculated at the value $\bar{c}_{0}=1.054$. Shapes $3-8$ are calculated for the constant value of the reduced volume $v=0.95$. The significance of the points $A, B, C, D$ and $E$ is described in the text. The shapes are determined by the methods described in Svetina and Zeks (1989) and Seifert et al. (1991).

\section{Discussion}

The problem of epithelial invagination has been analyzed on the basis of the mechanical properties of laminar membranes. In particular, it was assumed that the blastula wall can be described as a laminar structure. It was also considered that the system is laterally homogeneous, which would be so if blastula cells were identical. In real systems, cells differentiate during the blastula stage and this may cause the mechanical properties of the blastula wall to be laterally heterogeneous. However, certain general conclusions concerning the shape behavior of closed objects with laminated envelopes that were revealed by studying laterally homogeneous systems, e.g., the existence of shape classes and the bilayer couple mechanism of shape transformations, remain valid also for more complex systems with laterally heterogeneous envelopes. It is therefore an appropriate approach, to a first approximation, by which to study the shape behavior of closed monocellular sheets in terms of the shape behavior of simple vesicular objects that exhibit homogeneous envelopes. The discussion will be divided into two parts. Firstly we shall emphasize the general principles that govern the shape behavior of vesicular objects and which could thus serve as a framework for analysis of more complex monocellular systems. Secondly, we shall discuss the specific problems relating to shape transformations of closed monocellular sheets and attempt to relate the results to the mechanical aspects of the process of primary invagination in the blastula-gastrula transition of the sea urchin.

Shapes of vesicular objects can be conveniently characterized by two geometrical variables - the reduced volume $(v)$ and the reduced integral vesicle curvature $(\bar{c})$. Accordingly, the invaginated shape of the gastrula can be identified as one of the possible natural shapes of a vesicular object and the sphere as another. These two shapes correspond to two different sets of the two shape variables. The study of the blastula - gastrula transition can thus be centered on the question as to which mechanisms act to change values of these two variables. In general, there are different possible mechanisms. The reduced volume $v$ can decrease from the value 1 of the sphere either at constant envelope area, due to the loss of liquid enclosed by the blastula wall, or at constant volume, due to the increase of the area of this wall. Mechanisms to change the variable $\bar{c}$ can be based on those that affect the reduced spontaneous curvature $c_{0}$ and the reduced equilibrium integral vesicle curvature $\bar{c}_{0}$, the latter for example by differential changes of the equilibrium areas of different layers that constitute the blastula wall (Eq. 6). The pathway along which vesicle shapes transform from sphere to the invaginated gastrula shape in the $v-\bar{c}$ phase diagram is governed by the mechanical properties of the blastula wall. As has been shown, the basic elastic deformational modes needed to describe the shape behavior of closed laminar membranes are the area expansivity and the local and non-local bending. These deformational modes constitute the minimum necessary basis for the shape determination of closed laminar membranous systems. The same set of deformational modes is therefore a natural choice for describing, to a first approximation, the elastic behavior of the blastula wall.

One advantage of the suggested approach is that it makes it possible to study the mechanism of epithelial invagination in separate steps. There is a lower structural level at which an 
understanding of the specific intracellular and intercellular interactions should lead to derivation of the macroscopic elastic constants of the blastula wall. At a certain intermediate level, it is for instance possible to derive the elastic constants and the spontaneous curvature of a monocellular sheet in terms of the cortical tension of cells and the adhesion between them (Derganc, 2003). At a higher structural level, knowledge of the elastic constants suffices for the determination of shapes.

Because a blastula is initially spherical while a gastrula is not, the invagination process requires the blastula reduced volume to decrease. Thus, in the course of gastrulation, the system has to change in such a way as to cross the shape instability line shown in Figs. 2 and 3A. In the example treated (Fig. 3) the instability line was taken to be reached by decrease of the pressure difference across the blastula envelope. This is not an unrealistic possibility in view of the reported effects of the blastocoel osmotic pressure on the gastrulation process (Takata and Kominami, 2001). However, as is evident from Fig. 2, the instability line can also be reached by processes that would cause an increase of the reduced spontaneous curvature $c_{0}$ or of the reduced equilibrium integral vesicle curvature $\bar{c}_{0}$.

It has been predicted theoretically that in general the shape obtained after crossing the sphere instability line belongs to the class of axisymmetric shapes that also exhibit equatorial mirror symmetry. Gastrula shapes do not possess the latter symmetry, therefore in the course of subsequent shape transformations there has to be a symmetry breaking transition from symmetric (in our case oblate) shape to the asymmetric cup shape. As presented in Fig. 1, this transition can be continuous or discontinuous, depending on the reduced volume and on the ratio between the non-local and local bending constants. At reduced volumes that correspond to the reduced volume of a typical gastrula, phospholipid vesicles exhibit discontinuous shape transformations (Majhenc et al., 2004), whereas the gastrulation process proceeds as a series of continuous shape transformations. The difference in the behavior of these two vesicular objects must reflect physical differences in their envelopes. Each shape transformation, continuous or discontinuous, requires the rearrangement of the basic constituents of the envelope - phospholipid molecules in phospholipid membranes and cells in monocellular sheets. It is obvious that lipid molecules can rearrange their mutual positions much more easily than cells. It is anticipated that, under the conditions of continuous shape transformation, there is enough time for cells to adapt their mutual positions according to the requirements of slowly changing shapes but that, in the case of discontinuous shape transformations, such cell rearrangements cannot be accomplished. The requirement for continuous shape transformations poses some limitations on the mechanical properties of the blastula wall, for instance it has to have a value of the ratio $k_{r} / k_{c}$ that is at least one order of magnitude larger than the typical value for simple lipid membranes of around 3 (Waugh et al., 1992, Majhenc et al., 2004).

Blastula, at their high reduced volumes $v$, can transform their shape continuously from oblate axisymmetric that involves also equatorial mirror symmetry, into oblate shape without the latter symmetry, only if the ratio between the non-local and local bending $k_{r} / k_{c}$ of its wall is sufficiently large (Fig. 1). The question is whether epithelial sheets are adapted to fulfill this requirement. We consider the available data on the behavior of the blastula of the sea urchin and follow the idea about the role of apical layers in the blastula-gastrula transition, supported by the accumulated evidence supporting their indispensability for the occurrence of gastrulation. According to the gel swelling mechanism (Lane et al., 1993), the relevant layers of the sea urchin envelope are the hyaline layer, the apical lamina and the monocellular sheet (Fig. 4). The hyaline layer ( $\mathrm{HL}$ ) consists of a network of hyaline and other filaments. In general, filamentous networks are much less resistant to bending than to area extension. We therefore assume that the hyaline layer contributes to the envelope mechanics mainly by its area expansivity energy term (Eq. 1) and that its bending deformational mode can be neglected. The monocellular layer $(\mathrm{CL})$ is much thicker and therefore, in addition to the area expansivity contribution, it also exhibits resistance to bending. This layer is also the main candidate for nonzero spontaneous curvature of the blastula wall. The apical lamina $(A L)$ is the region between the hyaline layer and the monocellular sheet and is filled with protein gel. Its width appears to be under the control of the length of microvilli that keep the hyaline layer at a certain distance from the cell apical surfaces. When the gel is swollen by absorbed water, one can thus suppose that the increase of the lamina volume manifests itself in increased equilibrium area of the apical lamina $\left(A_{0, A L}\right)$. The mechanical properties of this lamina are equivalent to the mechanical properties of a confined water layer. Because water is practically incompressible, the effective area expansivity modulus of the apical lamina is very large and its bending modulus is practically zero.

The differences in the elastic behavior of different layers of the sea urchin blastula wall can be used as the basis for elucidating the mechanism of blastula invagination. The characteristic features of the described trilayer model of the blastula wall can be elucidated on the basis of Eq. 5 for the case where the apical lamina has a much larger area expansivity modulus than the other two layers. In such a case the neutral surface of the membrane coincides with the neutral surface of the apical lamina at its middle. The non-local bending constant can then be expressed as

$$
k_{r}=h_{H L}^{2} K_{H L}+h_{C L}^{2} K_{C L},
$$

where $h_{H L}$ and $h_{C L}$ are the distances from the neutral surface of the apical lamina to the neutral surfaces of the hyaline and monocellular layers, respectively (Fig. 4). The corresponding approximate equilibrium value of the integral vesicle curvature, $\bar{C}_{0}$ (see Eq. 6), is conveniently expressed as

$$
\bar{C}_{0}=\frac{\left(A_{0, H L}-A_{0, A L}\right)+p\left(A_{0, A L}-A_{0, C L}\right)}{h_{H L}+p h_{C L}},
$$

where $A_{0, H L}, A_{0, A L}$ and $A_{0, C L}$ are the equilibrium areas of the hyaline layer, apical lamina and monocellular sheet, respectively and the factor $p$ is given by

$$
p=\frac{h_{C L} K_{C L}}{h_{H L} K_{H L}} .
$$

Increase of the integral vesicle curvature $\bar{C}_{0}$ promotes evagination whereas its decrease promotes invagination. If swelling of the apical lamina (i.e. increasing of $A_{0, A L}$ ) is to cause the blastula invagination, $\bar{C}_{0}$ has to decrease as $A_{0, A L}$ increases. This can happen only if the value of the ratio $p(\mathrm{Eq} .12)$ is sufficiently small. The requirement for the described mechanism of blastula invagi- 


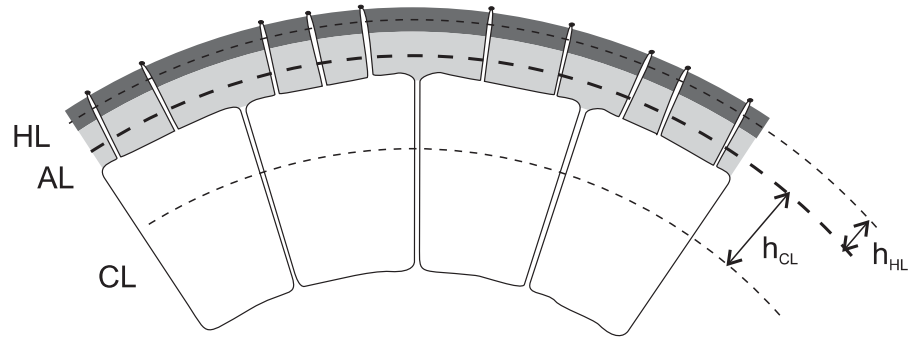

Fig. 4. Schematic representation of the laminar structure of the treated model for the blastula wall. The innermost layer is taken to be monocellular sheet (CL), in the middle is the apical lamina $(A L)$ and the outermost layer is the hyaline layer (HL). The dashed lines represent the positions of the layers' neutral surfaces. Since the apical lamina expansivity modulus is much larger than those of the other two, the neutral surface of the whole blastula wall is assumed to coincide with its neutral layer (bold dashed line). The distances of the neutral surfaces of HL and CL to the neutral surface of the wall are denoted by $h_{H L}$ and $h_{C L^{\prime}}$ respectively.

nation is thus that the area expansivity modulus of the monocellular layer is sufficiently small compared to the area expansivity modulus of the hyaline layer. This can be so, since experimental evidence shows that the stiffness of the extracellular matrix is much greater than that of the monocellular layer (Davidson et al., 1999). Also, the neutral surface of the monocellular layer should lie as close to the apical side of the cells as possible. It appears that the latter requirement was achieved in the evolution of monocellular epithelial sheets by positioning cell-cell junctions close to the apical sides. The bending constant of the monocellular layer is, to a first approximation, proportional to the area expansivity modulus $K_{C L}$, so that with the small value of this modulus it is also small. Taking into account these characteristics of the blastula wall, it follows that the ratio between non-local and local bending constants is given approximately by $k_{r} / k_{c}=K_{H L} h_{H L}{ }^{2} /$ $k_{c, C L}$. The area expansivity modulus $\left(K_{H L}\right)$ can be estimated from its elastic modulus $E_{H L}$ as $K_{H L} \approx E_{H L} d_{H L}$, where $d_{H L}$ is the thickness of the hyaline layer. $E_{H L}$ was determined to be of the order of $10^{4}$ Pa or more (Davidson etal., 1999) and $d_{H L}$ is approximately $1 \mu \mathrm{m}$. Therefore, we have $K_{H L} \geq 10^{4} \mu \mathrm{J} / \mathrm{m}^{2}$ and from $k_{r} / k_{c}$ we can estimate $k_{c} \geq 10^{-16} \mathrm{~J}$.

For interpretation of the blastula-gastrula shape transformation, a particularly important deformational mode is the non-local bending mode and the crucial parameter of the corresponding energy term is the equilibrium value of the integral vesicle curvature $\bar{C}_{0}$. As seen in Eq. 11, the value of this parameter depends to a large extent on the equilibrium areas of the layers that constitute the envelope. There are many physical and chemical means by which cells can control the magnitudes of these areas. The already discussed secretion of hygroscopic molecules into the apical lamina in the case of gastrulation (Lane et al., 1993) constitutes just one possible mechanism. It can be inferred that the processes causing differential changes of the equilibrium areas of membrane layers and consequent shape transformations are a suitable means for the transfer of chemical energy into mechanical work needed to drive the folding of monocellular sheets.

The elastic properties of the blastula wall described above support the model of epithelial invagination proposed by Lane et al. (1993). Our analysis implies that, for this mechanism to work, it is essential that the area expansivity modulus of the monocellular sheet be much smaller than the corresponding modulus of the hyaline layer. In the same sense it can also be suggested that within the proposed trilayer model of the blastula wall, exogastrulation (for this phenomenon see e.g. Hardin and Cheng, 1986) can be interpreted as the consequence of such changes in the values of parameters in the numerator of Eq. 11 that cause its second term to become larger than the first one.

In conclusion, this analysis provides a theoretical background to the mechanism of epithelial invagination in sea urchin gastrulation, based on swelling the apical lamina and in this way enlarging its area relative to the area of the hyaline layer (Lane $e t$ al., 1993). The treatment of the trilayer model of the blastula wall has indicated in particular the demands on the elastic properties of the monocellular layer for this mechanism to be operative. In this contribution we have especially emphasized the general shape behavior of vesicular objects as a possible framework for analysis of epithelial invagination. It is implied that the analysis of different possible mechanisms of this process could benefit if performed also from the perspective that some of the observed features of the invagination process are the consequence of general principles that shapes of vesicular objects obey.

\section{References}

BELOUSSOV, L.V. (1998). The Dynamic Architecture of a Developing Organism. An Interdisciplinary Approach to the Development of Organisms. Kluwer Academic Publishers, Dordrecht, Boston, London. pp. 1-238.

CANHAM, P.B. (1970). The minimum energy of bending as a possible explanation of the biconcave shape of the human red blood cell. J. Theor. Biol. 26: 61-81.

DAVIDSON, L.A., KOEHL, M.A.R., KELLER, R. and OSTER, G.F. (1995). How do sea urchins invaginate? Using biomechanics to distinguish between mechanisms of primary invagination. Development 121: 2005-2018.

DAVIDSON, L.A., OSTER, G.F., KELLER, R.E. and KOEHL, M.A.R. (1999) Measurements of mechanical properties of the blastula wall reveal which hypothesized mechanisms of primary invagination are physically plausible in the sea urchin Strongylocentrotus purpuratus. Dev. Biol. 209: 221-238.

DERGANC, J. (2003). Studij mehanizmov agregacije celic $v$ linearne in dvodimenzionalne strukture. Ph.D. Thesis, University of Ljubljana.

DEULING, H.J. and HELFRICH, W. (1976). The curvature elasticity of fluid membranes: a catalogue of vesicle shapes. J. Phys. France 37: 1335-1345.

ETTENSOHN, C.A. (1985). Mechanisms of epithelial invagination. Q. Rev. Biol. 60 : 289-307.

EVANS, E. and YEUNG, A. (1989). Apparent viscosity and cortical tension of blood granulocytes determined by micropipet aspiration. Biophys. J. 56: 151-160.

GUSTAFSON, T. and WOLPERT, L. (1967). Cellular movement and contact in sea urchin morphogenesis. Biol. Rev. 42: 442-498.

HARDIN, J. D. and CHENG, L. Y. (1986). The mechanism and mechanics of archenteron elongation during sea urchin gastrulation. Dev. Biol. 115: 490-501.

HEINRICH, V., SVETINA, S. and ZEKS, B. (1993). Non-axisymmetric vesicle shapes in a generalized bilayer-couple model and the transition between oblate and prolate axisymmetric shapes. Phys. Rev. E48: 3112-3123.

KELLER, R., DAVIDSON, L.A. and SHOOK, D.R. (2003). How we are shaped: The biomechanics of gastrulation. Differentiation 71: 171-205.

KOEHL, M.A.R. (1990). Biomechanical approaches to morphogenesis. Sem. Dev. Biol. 1: 367-378.

KOMINAMI, T and TAKATA, H. (2004). Gastrulation in the sea urchin embryo: A model system for analyzing the morphogenesis of a monolayered epithelium. Develop. Growth Differ. 46: 309-326.

LANE, M.C., KOEHL, M.A.R., WILT, F. and KELLER, R. (1993). A role for regulated secretion of apical extracellular matrix during epithelial invagination in the sea urchin. Development 117: 1049-1060. 
MAJHENC, J., BOZIC, B., SVETINA, S. and ZEKS, B. (2004). Phospholipid membrane bending as assessed by the shape sequence of giant oblate phospholipid vesicles. Biochim. Biophys. Acta 1664: 257-266.

MIAO, L., SEIFERT, U., WORTIS, M. and DOEBEREINER, H.G. (1994). Budding transitions of fluid-bilayer vesicles: the effect of the area difference elasticity. Phys. Rev. E49: 5389-5407.

NEWMAN, S. A. and COMPER, W. D. (1990). Generic physical mechanisms of morphogenesis and pattern formation. Development 110: 1-18.

ODELL, G.M., OSTER, G., ALBERCH, P. and BURNSIDE, B. (1980). The mechanical basis of morphogenesis. I. Epithelial folding and invagination. Dev. Biol. 85: 446-462.

OU-YANG, Z-C. and HELFRICH, W. (1989). Bending energy of vesicle membranes: General expressions for the first, second and third variation of the shape energy and applications to spheres and cylinders. Phys. Rev. A39:5280-5288.

SEIFERT, U. BERNDL, K. and LIPOWSKY, R. (1991). Shape transformations of vesicle-phase-diagram for spontaneous-curvature and bilayer-coupling models. Phys. Rev. A 44: 1182-1202.

SHEETZ, M.P. and SINGER, S.J. (1974). Biological membranes as bilayer couples. A molecular mechanism of drug-erythrocyte interaction. Proc. Natl. Acad. Sci. USA 71: 4457-4461.

SPIEGEL, E., HOWARD, L. and SPIEGEL, M. (1989). Extracellular matrix of sea urchin and other marine invertebrate embryos. J. Morph. 199: 71-92.

SVETINA, S. and ZEKS, B. (1989). Membrane bending energy and shape determi- nation of phospholipid vesicles and red blood cells. Eur. Biophys. J. 17: 101111.

SVETINA, S. and ZEKS, B. (1991). Mechanical behavior of closed lamellar membranes as a possible common mechanism for the establishment of developmental shapes. Int. J. Dev. Biol. 35: 359-365.

SVETINA, S. and ZEKS, B. (1992). The elastic deformability of closed multilayered membranes is the same as that of a bilayer membrane. Eur. Biophys. J.21:251255.

SVETINA, S. and ZEKS, B. (1996). Elastic properties of closed bilayer membranes and the shapes of giant phospholipid vesicles. In: (Lasic, D.D. and Barenholz, Y. Eds.). Handbook of Nonmedical Applications of Liposomes. CRC Press, Boca Raton. Vol. I: 13-42.

SVETINA, S. and ZEKS, B. (2002). Shape behavior of lipid vesicles as the basis of some cellular processes. Anat. Rec. 268: 215-225.

TAKATA, H. and KOMINAMI, T. (2001). Shrinkage and expansion of blastocoel affect the degree of invagination in sea urchin embryos. Zool. Sci. 18: 10971105.

VERHAS, J. (1997). Continuous shape transformations of liquid crystal membrane vesicles. J. Mol. Liquids 72: 337-346.

WAUGH, R.E., SONG J., SVETINA, S. and ZEKS, B. (1992). Local and non-local curvature elasticity in bilayer membranes by tether formation from lecithin vesicles. Biophys. J. 61: 974-982. 